\title{
Probability, rational single-case decisions and the Monty Hall Problem
}

\author{
Jan Sprenger
}

Received: 12 December 2007 / Accepted: 2 December 2008 / Published online: 23 December 2008 (C) The Author(s) 2008. This article is published with open access at Springerlink.com

\begin{abstract}
The application of probabilistic arguments to rational decisions in a single case is a contentious philosophical issue which arises in various contexts. Some authors (e.g. Horgan, Philos Pap 24:209-222, 1995; Levy, Synthese 158:139-151, 2007) affirm the normative force of probabilistic arguments in single cases while others (Baumann, Am Philos Q 42:71-79, 2005; Synthese 162:265-273, 2008) deny it. I demonstrate that both sides do not give convincing arguments for their case and propose a new account of the relationship between probabilistic reasoning and rational decisions. In particular, I elaborate a flaw in Baumann's reductio of rational single-case decisions in a modified Monty Hall Problem.
\end{abstract}

Keywords Probability · Decision Theory · Rationality · Monty Hall Problem

\section{Introduction: the Monty Hall Problem}

The application of probabilistic arguments to rational decisions in a single case is a non-trivial philosophical problem which arises in various contexts. This paper focuses on the validity of probabilistic arguments in the Monty Hall Problem and a variation thereof. The examination of this special case teaches us a general lesson about the normative force of probabilistic arguments.

The Monty Hall Problem arises in the following situation: You are a candidate in a TV show. You stand in front of three closed doors, one of them hiding a prize. The other two doors are empty. You do not know which door hides the prize and which doors are empty, but every door is equally likely to hide the prize. Monty Hall, the

J. Sprenger $(\bowtie)$

Tilburg Center for Logic and Philosophy of Science (TiLPS), Tilburg University, P.O. Box 90153, 5000 LE Tilburg, The Netherlands

e-mail: J.Sprenger@uvt.nl 
show moderator, asks you to guess a door. You make a guess, and afterwards Monty opens a door at random, but he will neither open the door you have chosen nor the door that hides the prize. So he will open an empty door. Then you have the opportunity to stick to your initial choice or to switch to the other closed door. You get the prize if you correctly guess the door where it is hidden. Which of the two actions should you take, given that you are rational, self-interested and perfectly know the rules of the game?

To answer that question, we have to compare the success expectations of the two strategies, by which I mean your rational single-case credence in the success of a certain strategy or action. Then, Bayes's theorem implies that you should switch to the other closed door. This may appear counterintuitive, but it is a sound recommendation: At the beginning, you have to bet on a particular door, but you would prefer to bet on two doors at once, in order to double your chances. In other words, you would like to guess that the prize is not behind a particular door. Such a guess is not allowed by the rules of the game, but the switching strategy indirectly provides it: Switching wins whenever the prize is not behind the originally picked door. The switching strategy has a success expectation of $2 / 3$, compared to $1 / 3$ when you stick to your initial choice: By maintaining their initial guess, stickers fix their success expectation on $1 / 3$ whereas switchers have a success expectation of $1-1 / 3=2 / 3$ : they win unless the initially chosen door was actually the winning one. I would like to call this the standard solution of the Monty Hall Problem. ${ }^{1}$

\section{Baumann's argument}

An objection to the standard solution of the Monty Hall Problem has recently been put forward by Baumann $(2005,2008)$. Baumann accepts the superiority of switching in the long run, but claims that epistemic single-case probabilities (success expectations) cannot be meaningfully applied to a modified version of the Monty Hall Problem. He infers that probabilistic arguments may not be applied to single cases of the original Monty Hall Problem either.

In Baumann's modified problem, there are two players instead of one. Both privately guess a door and submit their choice to Monty Hall. Again, Monty is not allowed to open any door that was selected by one of the players. In particular, when the players initially guess different doors and are both mistaken, Monty has no choice but to open the winning door. After the opening of a door, the players are asked whether they want to stick or to switch. In particular, they are allowed to switch to an open door with the prize behind it. Note that both players get the full prize if they succeed. Hence, there is no real competition between them. All this is common knowledge between the players, but no player knows the initial choice of the other player. Neither player has a preference for a specific door, and this is common knowledge, too.

Baumann goes on by comparing two different strategies: the conditional sticking and the conditional switching strategy. A player is a conditional sticker if she sticks to her initial choice unless the winning door is opened (then she switches to that door, of course). A player is a conditional switcher if she switches to the other closed door

$\overline{1}$ Cf. Bradley and Fitelson (2003). 
Table 1 The success expectations for conditional switching and sticking in the modified Monty Hall Problem for player $A$ if door 1 is the winning door

\begin{tabular}{lllll}
\hline$A$ 's choice & $B$ 's choice & $\begin{array}{l}\text { Opened } \\
\text { door }\end{array}$ & $\begin{array}{l}\text { Conditional } \\
\text { switching wins }\end{array}$ & $\begin{array}{l}\text { Conditional } \\
\text { sticking wins }\end{array}$ \\
\hline 1 & 1 & 2 or 3 & No & Yes \\
1 & 2 & 3 & No & Yes \\
1 & 3 & 2 & No & Yes \\
2 & 1 & 3 & Yes & No \\
2 & 2 & 3 & Yes & No \\
2 & 3 & 1 & Yes & Yes \\
3 & 1 & 2 & Yes & No \\
3 & 2 & 1 & Yes & Yes \\
3 & 3 & 2 & Yes & No \\
& & & $6 / 9$ & $5 / 9$ \\
\hline
\end{tabular}

unless the winning door is opened (then she switches to that door). ${ }^{2}$ So both strategies agree if Monty discloses the prize and differ in any other case.

Baumann argues that the conditional switching strategy is superior in the modified Monty Hall Problem. Assume without loss of generality that door 1 is the winning door. Then there are nine equiprobable cases as a function of the players' first choice (e.g. $A$ chooses door $1, B$ chooses door 2 , etc.). Table 1 compares the success expectations of conditional sticking and switching in the modified Monty Hall Problem, leading to a success expectation of $6 / 9$ for conditional switching, as opposed to 5/9 for conditional sticking. ${ }^{3}$ Note that the success expectations need not add up to 1 , unlike in the original Monty Hall Problem, since the strategies prescribe the same action whenever the winning door is opened. Thus, Baumann specifies his argument in a situation when Monty has opened an empty door: Two rows in Table 1 (the sixth and the eighth) cancel out and only seven — allegedly equiprobable — cases remain. The players count the remaining cases and assign success expectations of 4/7 to switching and $3 / 7$ to sticking. ${ }^{4}$ Assume now that at the beginning, player $A$ has picked door 1

\footnotetext{
2 Baumann's original terminology is modified sticking/switching strategy, but this may be misleading. I sometimes omit the qualification "conditional" when it is clear that an empty door has already been opened.

3 Cf. Baumann (2008, p. 267). Levy (2007, p. 143) objects to this chart, claiming that the success expectation of sticking is merely $3 / 9$ since sticking loses in the sixth and the eighth case. But obviously, by 'sticking' we refer to the conditional sticking strategy, cf. also Baumann (2008, p. 270). It could also be argued that 10 and not 9 cases must be distinguished: in the first line of Table 1, either door 2 or door 3 might be opened. But it is crucial for Baumann's argument that all relevant cases are equiprobable. That assumption is satisfied if we distinguish nine cases (the players choose independently and each door with equal probability) but not if we make further subdivisions.

${ }^{4}$ Cf. Baumann (2005, p. 72). Note that the opening of an empty door is evidence that the players initially picked the same door. In those cases, Monty would have opened an empty door with probability 1 whereas, if the players had picked different doors, he might have been forced to open the winning door. Thus, the new information favors some of the remaining seven cases over others and the equiprobability argument for a 4/7 success expectation of switching collapses (cf. Rosenhouse 2008, Sect. 7.5). But since Baumann's argument does not depend on specific probability values as long as the success expectation is not $1 / 2$, we can neglect this problem in the remainder.
} 
Table 2 Rational credences of player $A$ and $B$ in the given propositions, given that $A$ initially picks door $1, B$ initially picks door 2 and door 3 turns out to be empty

\begin{tabular}{lll}
\hline & $\begin{array}{l}\text { 'The other } \\
\text { door wins' }\end{array}$ & 'Door 2 wins' \\
\hline$A$ 's rational credence & $4 / 7$ & $4 / 7$ \\
$B$ 's rational credence & $4 / 7$ & $3 / 7$ \\
\hline
\end{tabular}

and player $B$ door 2. So Monty is forced to open door 3 which turns out to be empty. By conditioning on that information, both players arrive at the probabilities displayed in Table 2. The table reveals a difference in the rational credences $A$ and $B$ assign to the proposition 'door 2 wins'.

Now, the essential argument begins. We unanimously accept the principle of nonarbitrariness:

- if two agents' rational credences are fully determined by the available evidence and

- if they share all relevant information

then their rational credences should be the same.

Baumann thinks that this principle is violated with regard to the players' rational credences in the proposition 'door 2 wins' (cf. Table 2). So we have to check whether the premises of the Principle of Non-Arbitrariness are satisfied. The rules of the game and Monty's opening of the empty door 3 fully determine the players' rational credences, so the first premise is satisfied. Whether the second premise is also satisfied is more contentious. The only candidate for a relevant informational difference between the players is the privately made first guess which the other player cannot know. Baumann objects, however, the number of the originally picked door

"[...] does not matter at all when it comes to one's probabilistic reasoning about sticking and switching."

Hence, both players share the same relevant information and the premises of the Principle of Non-Arbitrariness are satisfied whereas the conclusion is violated, or so Baumann argues. This constitutes a reductio against the probabilistic argument for switching after the opening of the empty door 3 . While the standard argument for switching contends that in a long run of games, the unopened door will contain the prize more often than our initial choice, we do not have an argument for switching to door 2 in that individual game. Our probabilistic argument cannot guide rational decisions in the modified Monty Hall Problem without invoking arbitrariness. This threatens, of course, the general validity of probabilistic arguments in single cases, e.g. in the original Monty Hall Problem, since similar counterexamples might be found.

\section{Baumann's argument revisited}

There are several ways to argue against Baumann's reductio. A natural reply consists in claiming that the introduction of a second player changes the situation in a way that (conditional) switching is no longer superior to (conditional) sticking. This is the

\footnotetext{
5 Baumann (2005, p. 76).
} 
road taken by Ken Levy (2007). Baumann's reductio starts with $A$ picking door $1, B$ picking door 2 and Monty's opening of the empty door 3. Levy observes that this situation is completely symmetric with regard to door 1 and door 2, unlike in the original Monty Hall Problem (cf. Sect. 1). The point of the standard solution was the opportunity to bet against the initially chosen door by always switching to the other closed door, thus achieving a success rate of $2 / 3$. This is no longer possible in the modified problem because $B$ 's choice interferes with Monty's reaction to $A$ 's initial guess, and vice versa. Hence, the original argument for switching does no longer apply. Due to the complete symmetry between door 1 and door 2, neither sticking nor switching can have an advantage, or so Levy argues. Both players should assign success expectation $1 / 2$ to the remaining doors when an empty door has been opened. So Baumann's reductio-which requires that $A$ and $B$ assign different success expectations to door 2 (cf. Table 2)—does not get off the ground:

“So the proper conclusion is not Baumann's-i.e. that probabilities cannot meaningfully apply to single games [. . . . Rather, the proper conclusion is that a player has no reason to switch [...] because the presence of the second player changes the situation in such a way that the probabilities for the remaining closed doors are not $1 / 3$ and $2 / 3$ but rather $1 / 2$ and $1 / 2 . " 6$

If that argument worked, it would solve the problem. ${ }^{7}$ Unfortunately, the argument can be shown to fail. Let us distinguish two cases: either the players have chosen different doors or they have chosen the same door. In the first case, the situation is completely symmetric, as observed by Levy. The players' initial picks are sufficient to determine Monty's action, regardless of where the price is hidden. So Monty's action favors neither of the two remaining doors over the other. However, this argument from symmetry does not apply if the players initially pick the same door. Since their choices coincide, Monty acts like in the original Monty Hall Problem with only one player. Thus, there is a probabilistic argument for switching, as shown in Sect. 1. After an empty door has been opened, the players do not know which of the two situations holds, but they know that both are real options. Recall that switching is more likely to yield success in one of the two situations while the success expectations are equal in the other situation. Thus, the players should conclude that switching is a (weakly) dominant strategy and that it should be preferred to sticking. Hence, Levy's account of the problem is untenable. Unlike external observers, the players cannot know whether the other player has chosen a different door.

This reasoning gives an elegant and intuitive proof of the superiority of the conditional switching strategy: Switching is more promising than sticking when Monty opens an empty door, and otherwise, both strategies coincide. Still, this argument does not exhibit any flaw in Baumann's reductio. In what follows I cast the second

\footnotetext{
6 (Levy, 2007, p. 144). My own italics.

7 Some passages in Levy (2007, pp. 143-144) allow the interpretation that Levy intends the scope of his argument to be restricted to situations where the players choose different doors. However, this would be inconsistent with the above quote: Levy is interested in the players' reasons for switching and sticking. For their decisions, the players have — due to their uncertainty about the other player's choice - to take into account all possible cases, not only cases where different doors are picked. Such a restriction would deprive Levy's argument of its impact on rational decisions.
} 
premise of the Principle of Non-Arbitrariness into doubt by arguing that there are relevant informational differences between both players. As Baumann admits himself, everything hinges on this question.

The only sensible candidates for relevant private (=non-shared) information are the numbers of the originally picked doors. Baumann notices that each player could forget her original choice, but for playing the game after the opening of an empty door, it would be sufficient to tell Monty whether to switch or to stick. In this way, Baumann argues that each player's private information-her initial guess-is irrelevant to the success expectations of the two strategies. All 'relevant' information can be expressed in terms of 'the other door' and 'the original door'; and the numbers of the originally picked doors are irrelevant. ${ }^{8}$

However, there are two senses of 'relevant' in Baumann's argument that are not clearly set apart from each other: relevant ${ }_{1}$ for being able to play the (modified) Monty Hall game, relevant 2 for the assignment of rational credences to specific doors. Baumann's 'forgetting argument' (2005, p. 74) merely shows that the players' initial choice is not relevant ${ }_{1}$ - they assign the same credence to 'the other door wins'. But for his reductio, Baumann must aim at irrelevance 2 , i.e. the private information has to be irrelevant for a probability assignment to door 2 (cf. Table 2 and Baumann (2005, p. 72)). However, the players do not share all relevant 2 information: $A$ 's knowledge of her initial guess (door 1) licenses her to an inference along the lines of my proof above: If $B$ has picked door 2, the opening of door 3 delivers no evidence at all, but if $B$ has picked door 1 , too, the opening of door 3 is evidence in favor of door 2 . So door 2 is in total favored over door 1 . Vice versa, $B$ uses her private information (she started with door 2) to make an inference in favor of door 1 . Neither of the two inferences is available to the other player. Hence, the players have different bits of relevant 2 information and the Principle of Non-Arbitrariness is not violated, invalidating Baumann's conclusion.

Note further that there is nothing paradoxical about the deviant success expectations in Table 2: we are concerned with degrees of belief, and this takes us into an intensional context. An indexical expression such as 'the other door' may be replaced by a rigidly referring expression such as 'door 2' only if from the believer's perspective, the two expressions co-refer. Therefore player $A$ assigns the same success expectation to 'the other door' and 'door 2' whereas player $B$ assigns different success expectations. While the players rationally agree on the success expectation of 'the other door', they don't agree on that of 'door 2'; accordingly, relevance 2 does not imply relevance ${ }_{1}$.

Baumann's argument thus succumbs - and this is the main tenet of this sectionto an ambiguity concerning what is meant by the players' private information. We can happily concede that this information is not relevant ${ }_{1}$ while maintaining that it is relevant $t_{2}$-relevant to the success expectations of specific doors. Thus the second premise of the Principle of Non-Arbitrariness is not satisfied and Baumann's reductio does not go through.

In this section, we have rebutted Levy's argument that there is no advantage to conditional switching, proven the superiority of conditional switching, and pointed out

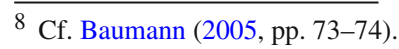


the crucial flaw in Baumann's reasoning. It remains to sketch the general relationship between probabilistic calculations and rational decisions.

\section{In defense of probabilistic arguments}

In his (2005), Baumann concludes that probabilistic arguments cannot guide rational decisions in single games (at least in certain scenarios) whereas they affect rational decisions in a sufficiently long run of games (see Baumann 2005, 77). Baumann does not demarcate the long versus the short run-i.e. it remains unclear whether a sufficiently long run consists of 5, 50, 500 or infinitely many Monty Hall games. So his conclusion remains vague. However, it is tempting to claim that rational credences about single cases have to match rational credences about the long run. Horgan (1995) establishes an 'isomorphism' between those types of credences "whenever certain known epistemic single-case probabilities [...] determine certain further epistemic single-case probabilities whose values one seeks to discover". 9 I am happy to grant his argument, but it rests on the meaningful applicability of some epistemic singlecase probabilities, and Baumann could deny that this premise is ever satisfied. So Horgan's argument begs the question. Although I share the commonsensical intuition that rational expectations in single and repeated games cannot differ (see also Levy 2007, 150), I feel the need to supplement my dismissal of Baumann's reductio by a positive account of the normative force of probabilistic arguments.

In his (2007), Levy claims that the players' rational success expectations are derived from the 'causal structure' of a Monty Hall game. This is a term already found in Moser and Mulder (1994) and Horgan (1995), so it deserves some attention. For Levy, "the causal structure of a Monty Hall game is the set of conditions that ultimately explains why sticking and switching have the probabilities that they do". ${ }^{10}$ To my mind, this analysis is empty (what are these conditions?) and therefore not helpful in resolving Baumann's challenge. On a more charitable reading, Levy (2007, p. 146) classifies those conditions into two groups: first, real facts as the fact that only one of the three doors hides the prize, and second, the information situation of the agent. For instance, at the beginning, she has no evidence in favor or against a specific door. According to Levy, this implies that the agent should set her success expectation for any specific door to $1 / 3$.

While I share Levy's conclusion, I have some reservations about his argument. First, the place of causality in the 'causal structure' of a Monty Hall game remains obscure. Rather, the symmetry of the agent's informational situation apparently plays the crucial role: If one has no evidence in favor of a specific door among three equallylooking doors, each door obtains a success expectation of $1 / 3$. Second, to claim that those credences are uniquely rational requires additional assumptions. On a minimal Bayesian account, credences are rational if and only if they are immune to Dutch Books and emerge from Bayesian Conditionalization (BC). So any credence that is neither vulnerable to a Dutch book nor violates (BC) is in principle admissible, and

\footnotetext{
${ }^{9}$ Horgan (1995, pp. 219-220).

${ }^{10}$ Levy (2007, p. 146).
} 
this applies even to a non-standard credence assignment as $(1 / 2,1 / 4,1 / 4)$ to the three doors.

There are two principal ways to avoid this consequence: First, we could invoke the Principle of Maximum Entropy (MaxEnt): We should set our credences to values that are as equivocal as possible, given our external constraints (e.g. background information). Levy's 'no further evidence' argument indicates the lack of such constraints on our credences and prompts an application of MaxEnt, leading to a uniquely rational credence of $1 / 3$ for each door. I doubt, however, that such a resolution is satisfactory. MaxEnt is an appealing, but also a very strong and controversial principle: In many situations we do not have precise and univocal information about the probability distribution which we try to elicit. MaxEnt nevertheless assigns definite, uniquely rational credences, blurring the distinction between risk and uncertainty (cf. Seidenfeld 1979).

The second way to ensure that each door gets $1 / 3$ credence at the beginning consists in endorsing the Principal Principle (PP, Lewis 1981). This is a move made by many Bayesians. The Principal Principle transfers known objective chances ("each door is equally likely to hide the prize") to rational single-case credences ("my degree of belief that door 1 wins is $1 / 3$ "). The game description posits such objective chances, e.g. in the Monty Hall Problem, it is assumed that the mechanism which assigns the prize to a specific door is not biased in favor of a particular door. Thereby we obtain definite single-case credences in the Monty Hall Problem, and consequently, we can base our rational decisions on these credences.

Three objections might be raised at that point: First, doesn't the Principal Principle presuppose the meaningful use of single-case probabilities and thus beg the question against Baumann? No. As Baumann himself writes:

"I argue that talk about winning by 'choosing door 1' or 'choosing door 2' [in a single game] does not make sense. However, I am not using this assumption as a premise [...]. The form of the argument is rather a reductio."11

Baumann's reductio being refuted, I do not see any evil in explicating how rational single-case credences are derived from objective chances. Indeed, none of Baumann's arguments is directed against (PP).

Second, (PP) could be rejected. But the consequences of such a move are not worth the evident rewards. Rejecting (PP) would destroy the link between known properties of the world (a coin is fair, an atom is likely to decay soon) and our corresponding beliefs. Counterexamples to (PP) have the form

(\&) "With $50 \%$ chance, a $P b^{210}$ atom will disintegrate in the next hour, but my rational credence (in the absence of additional information about the process) in that event is greater (or smaller) than $1 / 2 . "$

As Lewis (1981) pointed out, such assertions are evidently absurd-(PP) is required for calibrating our knowledge of the world with our degrees of belief (cf. Strevens 1999). Thus, (PP) should not be dismissed.

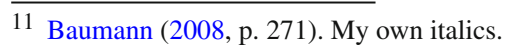


Third and last, it could be argued that in a Monty Hall Problem, we do not deal with genuinely objective chances-for instance, the players are no genuine randomizers when it comes to picking a door. There are no real objective chances which could transfer to rational credences, and consequently, (PP) cannot be applied. However, it is crucial to realize that the game description is idealized. We describe the game as if genuine chance processes were involved, in order to capture the relevant features of the game and to make the problem mathematically tractable. Inside this idealized model, we are supposed to act $a$ s if all processes were really chancy-a wisdom known to any poker or blackjack gambler who does probabilistic calculations. The modeling assumptions themselves can be controversial, but that concerns the external validity of our probabilistic model (does it apply to Monty Hall's TV show?), not the rationality of decisions inside this model.

Hence, the objections against (PP) can be rebutted. (PP) is indispensable for transferring properties of a probabilistic model to rational credences. Horgan and Levy's attempts to substantiate probabilistic arguments in single cases make more demanding assumptions and are therefore vulnerable to serious objections.

\section{Conclusions}

A lot of philosophers have used the Monty Hall Problem to derive far-reaching consequences for probabilistic reasoning. This paper has tried to eliminate some fallacies in the debate and to ground the normative force of probabilistic arguments. Below I summarize the principal points.

First, Baumann's attempt to argue against the normative force of probabilistic arguments in single cases fails. To succeed with his reductio, he requires that there be no relevant informational difference between the players. However, he does not distinguish two pertinent senses of 'relevant': namely, relevant ${ }_{1}$ for being able to play the game and relevant 2 for the success expectations of a particular door. Baumann's reductio would go through if he could show irrelevance 2 , but as I have argued, he

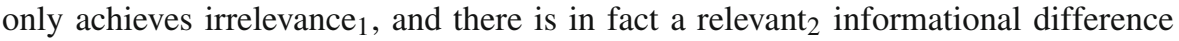
between the players. Therefore the Principle of Non-Arbitrariness is not violated, and Baumann's reductio does not get off the ground. Moreover, a simple, purely qualitative argument demonstrates the superiority of the conditional switching strategy.

Second, probabilistic arguments in single cases obtain their normative force from the Principal Principle. This account can be defended against the charge of being question-begging. In fact, it makes more parsimonious assumptions than Levy and Horgan's proposals.

I conclude that probabilistic arguments guide rational decisions in a single case and that the superiority of conditional switching in the modified Monty Hall Problem can be rescued.

Acknowledgements I would like to thank Peter Baumann, Filip Buekens, Stephan Hartmann, Vincent Hendricks, Sebastian Lutz, Jacob Rosenthal, Jonah Schupbach and two anonymous referees of this journal for helpful advice, discussion and suggestions. 
Open Access This article is distributed under the terms of the Creative Commons Attribution Noncommercial License which permits any noncommercial use, distribution, and reproduction in any medium, provided the original author(s) and source are credited.

\section{References}

Baumann, P. (2005). Three doors, two players and single-case probabilities. American Philosophical Quarterly, 42, 71-79.

Baumann, P. (2008). Single case probabilities and the case of Monty Hall: Levy's view. Synthese, 162, 265-273.

Bradley, D., \& Fitelson, B. (2003). Monty Hall, Doomsday and confirmation. Analysis, 63, $23-31$.

Horgan, T. (1995). Let's make a deal. Philosophical Papers, 24, 209-222.

Levy, K. (2007). Single case probabilities and the case of Monty Hall: Baumann's view. Synthese, 158, $139-151$.

Lewis, D. (1981) A subjectivist's guide to objective chance. In R. C. Jeffrey (Ed.), Studies in inductive logic and probability, pp. 263-293. Berkeley: University of California Press.

Moser, P. K., \& Hudson Mulder D. (1994). Probability in rational decision-making. Philosophical Papers, $23,109-128$.

Rosenhouse, J. (2008). The Monty Hall Problem. Book Manuscript, Oxford: Oxford University Press (forthcoming).

Seidenfeld, T. (1979). Why I am not an objective Bayesian; some reflections prompted by Rosenkrantz. Theory and Decision, 11, 413-440.

Strevens, M. (1999). Objective probabilities as a guide to the world. Philosophical Studies, 95, 243-275. 